\title{
Unit-Based Interprofessional Leadership Models in Six US Hospitals
}

\author{
Christopher S. Kim, MD, MBA ${ }^{1 *}$, Emmanuel King, MD², Jason Stein, $\mathrm{MD}^{3}$, Edmondo Robinson, $\mathrm{MD}^{4}$, \\ Mohammad Salameh, MD5, Kevin J. O’Leary, MD, MS ${ }^{6}$
}

\begin{abstract}
${ }^{1}$ Department of Internal Medicine, University of Michigan, Ann Arbor, Michigan; ${ }^{2}$ Division of General Internal Medicine, University of Pennsylvania School of Medicine, Philadelphia, Pennsylvania; ${ }^{3}$ Department of Medicine, Emory University School of Medicine, Atlanta, Georgia; ${ }^{4}$ Department of Medicine, Christiana Care Health System, Wilmington, Delaware; ${ }^{5}$ Department of Hospital Medicine, Trinity Health, St. Joseph Mercy Hospital, Ann Arbor, Michigan; ${ }^{6}$ Department of Hospital Medicine, Northwestern University, Chicago, Illinois.
\end{abstract}

The landscape of hospital-based care has shifted to place greater emphasis on improving quality and delivering value. In response, hospitals and healthcare organizations must reassess their strategies to improve care delivery in their facilities and beyond. Although these institutional goals may be defined at the executive level, implementation takes place at local sites of care. To lead these efforts, hospitals need to appoint effective leaders at the frontlines. Hospitalists are well poised to take on the role of the local clinical care improvement leader based on their experiences as direct frontline caregivers and their integral roles in hospitalwide quality and safety initiatives. A unit-based leadership model consisting of a medical director paired with a nurse manager has been implemented in several hospitals to function as an effector arm in response to the changing landscape of inpatient care. We provide an overview of this new model of leadership and describe the experiences of 6 hospitals that have implemented it. Journal of Hospital Medicine 2014;9:545-550. (C) 2014 Society of Hospital Medicine
Hospital-based care has become more complex over time. Patients are sicker, with more chronic comorbid conditions requiring greater collaboration to provide coordinated patient care. ${ }^{1,2}$ Care coordination requires an interdisciplinary approach during hospitalization and especially during transitions of care. ${ }^{3,4}$ In addition, hospitals are tasked with managing and improving clinical workflow efficiencies, and implementing electronic health records $(\mathrm{EHR})^{5}$ that require healthcare professionals to learn new systems of care and technology. Payment models have also started to shift toward an incentive and penalty-based structure in the form of value-based purchasing, readmission penalties, hospital-acquired conditions, and meaningful use. ${ }^{4,6}$

In response to these pressures, hospitals are searching for ways to reliably deliver quality care that is safe, effective, patient centered, timely, efficient, and equitable. ${ }^{7}$ Previous efforts to improve quality in the general medical inpatient setting have included redesign of the clinical work environment and new workflows through the use of checklists and "whiteboards" to enhance communication, patient-centered bedside rounds, standardized protocols and handovers, and integrated clinical decision support using health information technology. ${ }^{8-13}$ Although each of these care

\footnotetext{
${ }^{\star}$ Address for correspondence and reprint requests: Christopher S. Kim, MD, Department of Internal Medicine, University of Michigan, Ann Arbor, Michigan; Telephone: 734-647-2892; Fax: 734-615-8401; E-mail: seoungk@med.umich.edu

Additional Supporting Information may be found in the online version of this article.

Received: December 2, 2013; Revised: March 14, 2014; Accepted: March 23, 2014

2014 Society of Hospital Medicine DOI 10.1002/jhm.2200

Published online in Wiley Online Library (Wileyonlinelibrary.com).
}

coordination activities has potential value, integrating them at the unit level often remains a challenge. Some hospitals have addressed this challenge by establishing and supporting a unit-based leadership model, where a medical director and nurse manager work together to assess and improve the quality, safety, efficiency, and patient experience-based mission of the organization. ${ }^{14,15}$ However, there are few descriptions of this leadership model in the current literature. Herein, we present the unit-based leadership model that has been developed and implemented at 6 hospitals.

\section{MODELS OF UNIT-BASED LEADERSHIP}

The unit-based leadership model is grounded on the idea that culture and clinical care are products of frontline structure, process, and relationships, and that leaders at the site of care can have the greatest influence on the local work environment. ${ }^{16,17}$ The objective is to influence care and culture at the bedside and the unit, where care is delivered and where alignment with organizational vision and mission must occur. The concept of the inpatient unit medical director is not new, and hospitals in the past have recruited physician leaders to become clinical champions for quality improvement and help establish a collaborative work environment for physicians and unit-based staff. $^{18-22}$ These studies report on the challenges and benefits of incorporating a medical director to inpatient psychiatry or general care units, but do not provide specific details about the recruitment and responsibilities for unit-based dyad partnerships, which are critical factors for success on multidisciplinary inpatient care units.

There are several logistical matters to consider when instituting a unit-based leadership model. These include the composition of the leadership team, 
selection process of the leaders, the presence of trainees and permanent faculty, and whether the units are able to geographically cohort patients. Other considerations include a clear role description with established shared goals and expectations, and a compensation model that includes effort and incentives. In addition, there should be a clearly established reporting structure to senior leadership, and the unit leaders should be given opportunities for professional growth and development. Table 1 provides a summary overview of 6 hospitals' experiences to date.

\section{DISCUSSION}

In reviewing our 6 organization's collective experiences, we identified several common themes and some notable differences across sites. The core of the leadership team was primarily composed of the medical director and nurse manager on the unit. Across all 6 organizations, medical directors had a portion of their effort supported for their leadership work on the unit. Leadership development training was provided at all of our sites, with particular emphasis on quality improvement (QI) methods such as Six-Sigma, Lean, or Plan, Do, Study, Act (PDSA). Additional leadership development sessions were provided through the organization's human resources or affiliated university. Common outcome measures of interest include patient satisfaction, interdisciplinary practice, and collaboration on the unit, and some hospital-acquired condition measures. Last, there is a direct reporting relationship to a chief or senior nurse or physician leader within each organization. These commonalities and variances are further detailed below.

\section{Establishing the Unit-Based Leadership Model}

The composition of the unit-based leadership model in our 6 organizations is predominantly a dyad partnership of medical directors and nurse managers. Although informal physician-nurse collaborative practices have likely been in existence at many hospitals, formalizing this dyad partnership is an important step to fostering collaborative efforts to improve quality of care. It is also essential for hospital leadership to clearly articulate the need for this unit-based leadership model. Whether the motivation for change is from a previously untenable practice environment, or part of an ongoing improvement program, the model should be presented in a manner that supports the organization's commitment to improve collaborative practices for better patient care. One of our 6 hospitals initiated this leadership model based on troubling relationships between nurses and physicians on some of their inpatient care units, which threatened to stall the organization's Magnet application. Implementation of the leadership model at the unit level yielded improvements in nurse-physician interactions, patient satisfaction, and staff turnover. ${ }^{15,23}$ Another of the hospitals first evaluated why a previous attempt at this model did not deliver the intended outcomes, and redesigned the model based on its analysis. ${ }^{14}$

Across all of the organizations featured here, a common driver behind the adoption of the unit-based leadership model was to bridge the divide between physician services and nursing and other allied health providers. We found that many of the physicians routinely had patients on multiple units, limiting the quantity and quality of collaborative practices between unit-based staff and physician teams. The unit-based dyad leaders are ideally positioned to build and foster a culture of collaboration, and our organizations have been inclusive to ensure the participation of a multidisciplinary group of providers, including representatives from pharmacy, environmental services, physical therapy, respiratory therapy, social work, case management, and nutrition at leadership meetings or in daily patient-care discussions. In addition, 2 of the organizations have added quality improvement specialist/project managers to their teams to support the physician-nurse manager leaders on the unit.

\section{Selection Process and Professional Development}

The traditional approach to hiring a physician leader or a nurse manager has been an isolated process of drafting a job description for each position and hiring within their respective departments. For the dyad partnership to be successful, there should be established goals and expectations that require shared responsibilities between the 2 partners, which should guide the selection of these leaders. Other leadership attributes and essential character traits that should be modeled by the unit-based leaders include good communication skills, respect among coworkers, and a collaborative approach to decision making and action. In addition, both physician leaders and nurse managers in these roles should have the ability to take a system's view, recognizing that within the complex network of healthcare providers and processes on their unit, these elements interact with each other, which lead to the outcomes achieved on their units. $^{24,25}$ Table 2 lists some general shared responsibilities, highlighting specific activities that can be used to achieve the established outcomes. As the unit's dyad leadership works together to address these shared responsibilities, they should keep their sights focused on the overall strategic goals of the healthcare organization. Bohmer has defined 4 habits of the highvalue healthcare organization that in turn can be reflected through the inpatient unit leadership model to capture these activities at the local level: (1) planning care for specific patient populations, (2) microsystem design, (3) measurement and oversight, and (4) self-study. ${ }^{26}$ In determining specific shared responsibilities for each dyad partner, it is important for these leaders to understand the clinical microsystem of their unit such as their patient population, interdisciplinary 


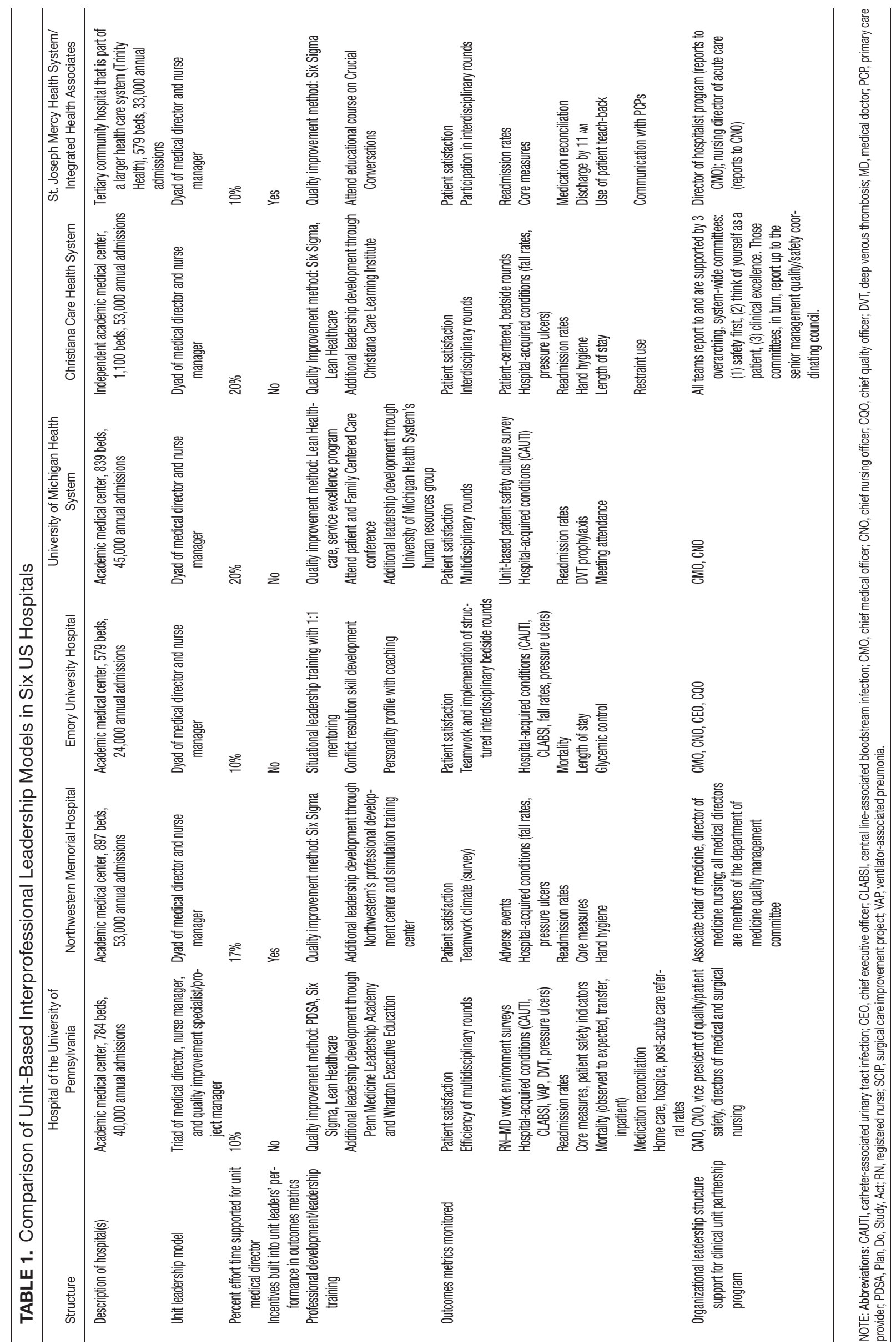




\begin{tabular}{|c|c|}
\hline $\begin{array}{l}\text { TABLE 2. General } \\
\text { Examples of Speci } \\
\text { Dyad Leadership }\end{array}$ & $\begin{array}{l}\text { ed Responsibilities With } \\
\text { ctivities Between the Unit }\end{array}$ \\
\hline $\begin{array}{l}\text { General Shared Responsi- } \\
\text { bilities of Physician and } \\
\text { Nurse Unit Directors }\end{array}$ & Examples of Specific Activities \\
\hline $\begin{array}{l}\text { Serve as management partners to } \\
\text { enhance culture of the unit }\end{array}$ & $\begin{array}{l}\text { Co-craft and deliver consistent leadership message } \\
\text { Co-establish and enforce unit processes and protocols } \\
\text { Co-lead recruitment and retention efforts } \\
\text { Co-orient trainees and faculty rotating through unit } \\
\text { Co-educate on the management of common medical } \\
\text { and surgical conditions } \\
\text { Facilitate interstaff conflict resolution sessions } \\
\text { Regular leadership meetings }\end{array}$ \\
\hline $\begin{array}{l}\text { Actively manage unit processes } \\
\text { and outcomes }\end{array}$ & $\begin{array}{l}\text { Quality: improve core quality measure performance } \\
\text { Safety: improve culture of patient safety within the unit } \\
\text { as measured by surveys and incident reporting } \\
\text { systems } \\
\text { Efficiency: reduce unnecessary length of stay and vari- } \\
\text { ability in resource use } \\
\text { Patient experience: focus on improving patient-family } \\
\text { experience with targeted outcomes in patient expe- } \\
\text { rience metrics (eg, HCAHPS) } \\
\text { Education: develop trainee and staff clinical and team- } \\
\text { work competencies }\end{array}$ \\
\hline $\begin{array}{l}\text { Continuous process improvement } \\
\text { initiatives (eg, PDSA cycles) }\end{array}$ & $\begin{array}{l}\text { Improve the discharge transitions process, tailoring the } \\
\text { process to each individual patient's identified risk } \\
\text { factors } \\
\text { Focus improvement efforts on reduction in specific hos- } \\
\text { pital acquired conditions such as CAUTI, VTE, } \\
\text { CLABSI, pressure ulcers, falls } \\
\text { Measure, analyze, reassess, and improve in all } \\
\text { described areas of shared responsibilities } \\
\text { Perform unit level chart reviews to evaluate readmis- } \\
\text { sions and LOS and identify improvement } \\
\text { opportunities }\end{array}$ \\
\hline
\end{tabular}

NOTE: Abbreviations: CAUTI, catheter-associated urinary tract infection; CLABSI, central line-associated blood stream infection; HCAHPS, Hospital Consumer Assessment of Healthcare Providers and Systems; LOS, length of stay; PDSA, Plan, Do, Study, Act; VTE, venous thromboembolism.

care team, approach to process improvement, and performance patterns over time. ${ }^{27}$

In our collective experience, the dyad leaders bring passion and commitment to improving care; however, many (the medical directors in particular) have minimal prior formal training in leadership, quality improvement, or hospital management. Recognizing that unit leaders require specialized knowledge and skills, each of our organizations has enrolled unit medical directors and nurse managers in leadership development courses or educational programs. Many healthcare organizations have become more grounded in a QI methodology including Six-Sigma, Lean Healthcare, PDSA, and other scientifically based methods, and the unit-based leaders should receive advanced training in the preferred methods of their institution. Additional training in quality improvement, patient safety, and physician leadership can also be obtained through supplemental coursework specifically designed to train hospital leaders, with some programs leading to a certification or additional credentials. ${ }^{28}$
Beyond such formal educational opportunities, hospitals should not overlook the opportunity to learn from and share experiences with the other dyad leadership units within the hospital. One of the organizations described here holds monthly meetings with all of the unit dyad leaders, and 2 other organizations conduct quarterly meetings to share experiences and best practices related to specific improvement initiatives in a learning network model. Those units with more experience in specific initiatives are asked to share their lessons learned with others, as well as support each other in their efforts to collectively meet the strategic goals of the hospital.

\section{Time and Organizational Support}

In addition to leadership development, hospitals and the clinical department leadership need to support the medical directors with dedicated time away from their usual clinical duties. Some organizations in this report are providing up to $20 \%$ effort for the medical director's unit-based leadership work; however, there is some variation in practice with regard to physician effort across sites. The University of Pennsylvania has a smaller effort support at $10 \%$; however, some of that effort differential may be offset through the allocation of the quality improvement specialist/project manager assigned to work with the medical director and nurse manager dyad. St. Joseph Mercy Hospital also has a lower allocation, as there is additional financial compensation for the role that is at risk and not included in this $10 \%$ allocation.

It is also important to assure that the medical directors have institutional support to carry out their work in partnership with their nursing leadership. The 6 health systems described here report that although most of the physicians have appointments within a physician group or clinical department, there is hospital leadership oversight from a chief medical, nursing, or operating officer. This organizational structure may be an important aspect of the model as the unit-based leaders seek to align their efforts with that of the hospital. Further, this form of organizational oversight can ensure that the unit leaders will receive timely and essential unit- and hospital-based performance measures to manage local improvement efforts. These measures may include some components of patient experiences as reported in the Hospital Consumer Assessment of Healthcare Providers and Systems survey, readmission rates, hospital-acquired condition rates, length of stay, observed to expected mortality rates, and results of staff satisfaction and safety culture surveys. As highlighted by several studies and commentaries, our collective experiences also identified interdisciplinary teamwork, collaboration, and communication as desirable outcome measures through the unit-based leadership structure. ${ }^{21,22,24,29,30}$ The medical director and nurse manager dyads can prioritize their 
improvement efforts based on the data provided to them, and mobilize the appropriate group of multidisciplinary practitioners and support staff on the unit.

\section{OTHER CONSIDERATIONS}

Other infrastructure variables that may increase the effectiveness of the unit leadership dyad include unitbased clinical services (geographic localization), engaging the frontline team members in the design and implementation of change innovations, a commitment to patient and family centered practices on the unit, and enhancing clinical workflow through the support of EHR functions such as concurrent documentation and provider order entry. Geographic localization, placing the fewest possible clinical service providers on the unit to work alongside unit-based staff, allows for a cohesive interdisciplinary unit-based team to develop under the dyad leadership, and has been shown to improve communication practices. ${ }^{9,31}$ Beyond geographic localization of patients, it is critical to ensure team members are committed to the changes in workflow by directly involving them through the design and implementation of new models of care taking place on the unit. This commitment starts from the top senior nurse and physician leaders in the organization, and extends to the unit-based dyad partners, and down to each individual interdisciplinary team member on the unit. ${ }^{1}$ Thus, it is critical to clarify roles and responsibilities and how team members on the unit will interact with each other. For some situations, conflict management training will be helpful to the unit-based leaders to resolve issues. To appreciate potential barriers to successful rollout of this unit leadership model, a phased implementation of pilot units, followed by successive waves, should be considered. Many of the units that instituted unit-based interdisciplinary team rounds solicited and implemented direct feedback from frontline team members in efforts to improve communication and be more patient centered. Conversely, there are also likely to be situations where the unitbased leaders will be confronted with hindrances to their unit-based collaborative improvement efforts. To help prepare the dyad leaders, many of our unit-based leaders have received specific training on how to coach and conduct difficult conversations with individuals who have performance gaps or are perceived to be hindering the progress of the unit's work. These crucial negotiation skills are not innate among most managers and should be explicitly provided to new leaders across organizations.

The goals and merits of patient- and familycentered care (PFCC) have been well described. ${ }^{32-34}$ Organizational support to teach and disseminate PFCC practices throughout all settings of care may help the leadership dyads implement rounding strategies that engage all staff, patients, and family members throughout the hospital course and during the transitions out of the hospital.

Clinical workflow has become heavily dependent on the EHR systems. For those organizations that have yet to adopt a particular EHR system, the leadership dyads should be involved throughout the EHR design process to help ensure that the technological solutions will be built to assist the clinical workflow, and once the system has been built, the leadership dyad should monitor and enhance the interface between workflow and EHR system so that it can support the creation and advancement of interdisciplinary plans of care on the unit.

\section{CONCLUSION}

The care of the hospitalized patient has become more complex over time. Interdisciplinary teamwork needs to be improved at the unit level to achieve the strategic goals of the hospital. Although quality improvement is an organizational goal, change takes place locally. Physician leaders, in partnership with nurse managers, are needed now more than ever to take on this task to improve the hospital-care experience for patients by functioning as the primary effector arms for changing the landscape of hospital-based care. We have described characteristics of unit-based leadership programs adopted across 6 organizations. Hospitalists with clinical experience as the principal providers of inpatient-based care and quality improvement experience and training, have been key participants in the development and implementation of the local leadership models in each of these hospital systems. We hope the comparison of the various models featured in this article serves as a valuable reference to hospitals and healthcare organizations who are contemplating the incorporation of this model into their strategic plan.

\section{References}

1. McIntosh N, Meterko M, Burgess JF Jr, et al. Organizational predictors of coordination in inpatient medicine [published online ahead of print February 26, 2014]. Health Care Manage Rev. doi: 10.1097/ HMR.0000000000000004.

2. Deb P. Trends in case-mix in the medicare population. Paper presented at: American Hospital Association, Federation of American Hospitals, Association of American Medical Colleges; http://www. aha.org/content/00-10/100715-CMItrends.pdf. July 15, 2010.

3. Williams MV. A requirement to reduce readmissions: take care of the patient, not just the disease. JAMA. 2013;309(4):394-396.

4. VanLare JM, Conway PH. Value-based purchasing-national programs to move from volume to value. N Engl J Med. 2012;367(4): 292-295.

5. Medicare and Medicaid programs; electronic health record incentive program. Final rule. Fed Regist. 2010;75(144):44313-44588.

6. Shrank W. The Center for Medicare and Medicaid innovation's blueprint for rapid-cycle evaluation of new care and payment models. Health Aff (Millwood). 2013;32(4):807-812.

7. Institute of Medicine. Crossing the Quality Chasm: A New Health System for the 21st Century. Washington, DC: National Academies Press; 2001.

8. O'Leary K, Wayne D, Haviley C, Slade M, Lee J, Williams M. Improving teamwork: impact of structured interdisciplinary rounds on a medical teaching unit. J Gen Intern Med. 2010;25(8):826-832.

9. O'Leary K, Wayne D, Landler M, et al. Impact of localizing physicians to hospital units on nurse-physician communication and agreement on the plan of care. J Gen Intern Med. 2009;24(11):1223-1227. 
10. Lau F, Kuziemsky C, Price M, Gardner J. A review on systematic reviews of health information system studies. I Am Med Inform Assoc. 2010;17(6):637-645.

11. Sehgal NL, Green A, Vidyarthi AR, Blegen MA, Wachter RM. Patient whiteboards as a communication tool in the hospital setting: a survey of practices and recommendations. J Hosp Med. Apr 2010;5(4): 234-239.

12. Henneman EA, Kleppel R, Hinchey KT. Development of a checklist for documenting team and collaborative behaviors during multidisciplinary bedside rounds. J Nurs Adm. 2013;43(5):280-285.

13. O'Leary KJ, Boudreau YN, Creden AJ, Slade ME, Williams MV. Assessment of teamwork during structured interdisciplinary rounds on medical units. J Hosp Med. 2012;7(9):679-683.

14. Kim CS, Calarco M, Jacobs T, et al. Leadership at the front line: a clinical partnership model on general care inpatient units. Am J Med Qual. 2012;27(2):106-111.

15. Rich VL, Brennan PJ. AHRQ health care innovations exchange: improvement projects led by unit-based teams of nurse, physician, and quality leaders reduce infections, lower costs, improve patient satisfaction, and nurse-physician communication. Available at: http://www. innovations.ahrq.gov/content.aspx?id=2719. Published April 14, 2010. Accessed November 26, 2011.

16. Huber TP, Godfrey MM, Nelson EC, Mohr JJ, Campbell C, Batalden PB. Microsystems in health care: part 8. Developing people and improving work life: what front-line staff told us. Jt Comm J Qual Saf. 2003;29(10):512-522.

17. Batalden PB, Nelson EC, Mohr JJ, et al. Microsystems in health care: part 5. How leaders are leading. Jt Comm J Qual Saf. 2003;29(6): 297-308.

18. Leibenluft E, Summergrad P, Tasman A. The academic dilemma of the inpatient unit director. Am J Psychiatry. 1989;146(1):73-76.

19. Pardini-Kiely K, Greenlee E, Hopkins J, Szaflarski NL, Tabb K. Improving and sustaining core measure performance through effective accountability of clinical microsystems in an academic medical center. Jt Comm J Qual Patient Saf. 2010;36(9):387-398.

20. Malloy E, Butt S, Sorter M. Physician leadership and quality improvement in the acute child and adolescent psychiatric care setting. Child Adolesc Psychiatr Clin N Am. 2010;19(1):1-19; table of contents.
21. Vazirani S, Hays RD, Shapiro MF, Cowan M. Effect of a multidisciplinary intervention on communication and collaboration among physicians and nurses. Am J Crit Care. 2005;14(1):71-77.

22. Clark RC, Greenawald M. Nurse-physician leadership: insights into interprofessional collaboration. J Nurs Adm. 2013;43(12):653-659.

23. The Advisory Board. University of Pennsylvania Health System pilots unit clinical leadership model to spur quality gains. Nurs Exec Watch. 2008;9(2):4-6.

24. Berwick DM, Nolan TW. Physicians as leaders in improving health care: a new series in Annals of Internal Medicine. Ann Intern Med. 1998;128(4):289-292.

25. Nolan TW. Understanding medical systems. Ann Intern Med. 1998; 128(4):293-298.

26. Bohmer RM. The four habits of high-value health care organizations. N Engl J Med. 2011;365(22):2045-2047.

27. Nelson EC, Batalden PB, Huber TP, et al. Microsystems in health care: Part 1. Learning from high-performing front-line clinical units. Jt Comm J Qual Improv. 2002;28(9):472-493.

28. Myers JS, Tess A, Glasheen JJ, et al. The quality and safety educators academy: fulfilling an unmet need for faculty development. Am J Med Oual. 2014;29(1):5-12.

29. Clemmer TP, Spuhler VJ, Berwick DM, Nolan TW. Cooperation: the foundation of improvement. Ann Intern Med. 1998;128(12 pt 1): 1004-1009.

30. Nancarrow SA, Booth A, Ariss S, Smith T, Enderby P, Roots A. Ten principles of good interdisciplinary team work. Hum Resour Health 2013;11(1):19.

31. Singh S, Tarima S, Rana V, et al. Impact of localizing general medical teams to a single nursing unit. J Hosp Med. 2012;7(7):551-556.

32. DiGioia AM III, Fann MN, Lou F, Greenhouse PK. Integrating patient- and family-centered care with health policy: four proposed policy approaches. Qual Manag Health Care. 2013;22(2):137-145.

33. Philibert I, Patow C, Cichon J. Incorporating patient- and familycentered care into resident education: approaches, benefits, and challenges. J Grad Med Educ. 2011;3(2):272-278.

34. Smith M, Saunders R, Stuckhardt L, McGinnis JM, eds. Best Care at Lower Cost: The Path to Continuously Learning Health Care in America. Washington, DC: National Academies Press; 2012. 